\section{ELISA goes subfemtomolar}

\section{By Kai-Jye Lou, Staff Writer}

Many proteins involved in cancer, neurological diseases and the early stages of infection are thought to be present in patient serum at concentrations well below the detection limit of current ELISA tests. Quanterix Corp. is looking to change that with its single-molecule array technology, which the company has shown can boost the sensitivity and detection limit of ELISA-based protein detection by over 1,000-fold compared with those of commercially available assays. ${ }^{1}$

Quanterix is using its single-molecule array (SiMoA) technology to detect and quantify protein biomarkers in the blood that are present at subfemtomolar $\left(<10^{-15} \mathrm{M}\right)$ concentrations. Next year, the company plans to launch its lead diagnostic as a Clinical Laboratory Improvement Amendments (CLIA)-certified test to detect prostate-specific antigen (KLK3; PSA) in patient serum.

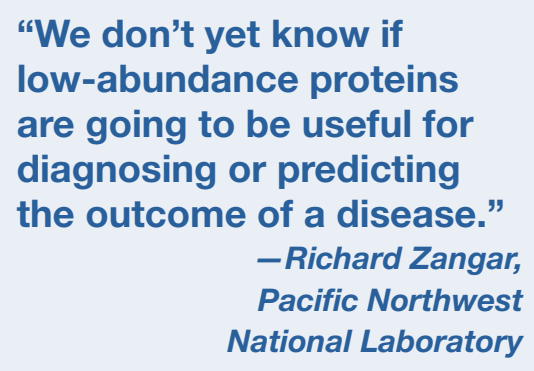

detection limit of about $3 \times 10^{-12} \mathrm{M}$, and the most sensitive commercially available ELISA for TNF- $\alpha$ had a detection limit of about $2.1 \times 10^{-14} \mathrm{M}$.

In serum samples taken from 30 prostate cancer patients who had undergone radical prostatectomy, the SiMoA-based assay detected PSA at concentrations as low as $4 \times 10^{-16} \mathrm{M}$.

"The ability to measure protein at these levels could allow for earlier diagnosis and disease treatment," said Duffy, who was corresponding author on the paper. "We showed we were able to detect and quantify up to 1,000-fold differences in PSA levels in blood samples taken from radical prostatectomy patients, all of whom had been told that they had undetectable PSA levels as measured by standard commercial assays."

Duffy noted that the SiMoA assays should be compatible with any protein as long as ELISA reagents are available. "If you have reagents that work well for a protein in standard ELISA, then it's a simple process of taking them and incorporating them into our system," he told SciBX.

"Having the ability to detect low-abundance proteins is important as these proteins are likely to be much more sensitive and specific markers for a particular disease than high-abundance proteins," said Richard Zangar, a staff scientist in cell biology and biochemistry at the Pacific Northwest National Laboratory.

He noted that earlier disease detection "could also facilitate the evaluation of new treatments that may not be effective at later stages of a disease."

"It may be a fair assumption that new protein biomarkers will be present at very low concentrations and that effective measurement and clini-

"The key advance is that we are using our single-molecule array technology to improve by orders of magnitude the sensitivity limits of protein detection using standard ELISA reagents," said David Duffy, senior director of platform research at Quanterix. "This technology allows us to digitally quantify a protein in blood, which has not been done before."

Sensitivity is the smallest change in concentration that can be detected using a particular approach. Detection limit is the lowest concentration that can be detected. Both are important because a change in serum levels of a biomarker may signify disease, whereas in other cases the mere presence of the biomarker is disease relevant.

SiMoA-based assays use standard immunoassay reagents, like antibodies and chemical labels, to capture and label single molecules of a protein on beads. Beads with or without single, labeled protein molecules are then loaded into an array containing 50,000-500,000 wells that act as femtolitersized reaction chambers. Due to their small size, wells containing a single bead with the protein of interest will generate a fluorescence signal above background levels and thus can be individually counted (see Figure 1, "ELISA using single-molecule array technology").

In a paper published in Nature Biotechnology, company researchers reported that assays using its technology detected human PSA and tumor necrosis factor- $\alpha$ (TNF- $\alpha$ ) that had been added to bovine serum. The detection limits for each protein were $2 \times 10^{-16} \mathrm{M}$ and $6 \times 10^{-16} \mathrm{M}$, respectively. In contrast, a commercially available immunoassay for PSA had a cal exploitation will depend heavily on the availability of highly sensitive assays," said Larry Kricka, director of general chemistry and the critical care laboratory and a professor of pathology and laboratory medicine at the University of Pennsylvania as well as a member of Quanterix's scientific advisory board. "This new single-molecule array-based technology could fulfill this unmet need.”

\section{The early bird}

Zangar agreed that SiMoA could open new areas of research involving low-abundance proteins but cautioned that the clinical utility of such proteins and the ability to detect them has not been established.

"We don't yet know if low-abundance proteins are going to be useful for diagnosing or predicting the outcome of a disease," he said. "They will also need to prove that the ability to detect low-abundance proteins will be clinically useful and beneficial to the patient."

Kricka thinks that upcoming challenges for the company's approach "will center on the development of the appropriate instrumentation that would provide clinical laboratories with the requisite level of automation and throughput."

A further challenge, he said, is to predict which tests or types of tests would need or benefit from the ultra-high sensitivity provided by the SiMoA technology.

Duffy said that "within the year, we plan to have a prototype system 


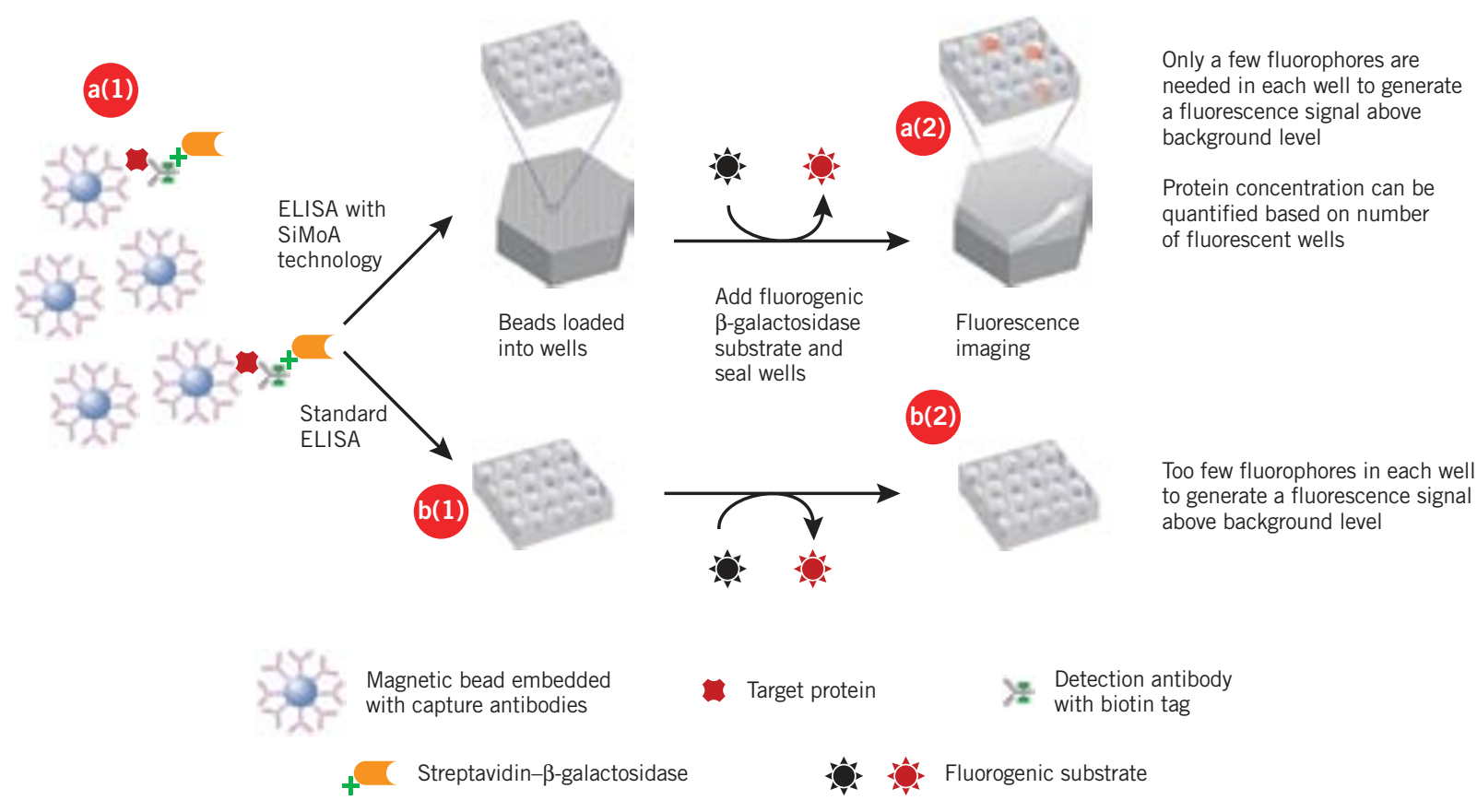

Figure 1. ELISA using single-molecule array technology. Immunoassays that use Quanterix Corp.'s single-molecule array (SiMoA) technology can detect and quantify proteins at concentrations below the detection limit of standard ELISA. The array contains 50,000-500,000 wells that act as femtoliter-sized reaction chambers.

Single protein molecules are captured onto beads embedded with capture antibodies for the target protein. Detection antibodies and streptavidin- $\beta$-galactosidase, which acts as the enzyme label, form an immunocomplex with the captured target protein. Since the target protein is present at very low concentrations, most beads will not capture any molecules of the target protein, and the beads that do should have only captured one [a(1)].

The beads are then loaded into the array of femtoliter-sized wells. Next, a fluorogenic $\beta$-galactosidase substrate is added and the array is sealed. Because the volume of each well is very small, few fluorophores per well are needed to generate a fluorescence signal above background levels. Wells containing a bead that captured a single target protein molecule will emit a detectable fluorescence signal [a(2)]. Protein concentration is quantified by counting the number of wells emitting the signal.

If the process were repeated using standard ELISA, the beads would be loaded into wells with volumes much larger than those used in the SiMoA [b(1)]. Because the volume in each well is now greater, more fluorophores per well are needed to generate a fluorescence signal above background levels. Thus, it may not be possible to detect and quantify a target protein when very low concentrations are present [b(2)].

that would have the degree of automation and throughput capacity that is needed for a clinical immunoanalyzer. To demonstrate the diagnostic and prognostic benefits of SiMoA tests, we are working closely with key opinion leaders in the urology and neurology fields to test high-quality clinical samples in well-controlled retrospective and prospective studies."

Quanterix is focused on developing a CLIA-certified test using SiMoA to measure PSA postsurgery. "It could be possible to use such a test to tell patients with extremely low PSA levels that they are essentially cured and also give patients with increasing PSA levels an earlier alert that they may need to go back for secondary treatment," said Duffy.

He said the company is running retrospective studies to determine if there are prognostic and diagnostic benefits to detecting differences in low PSA levels postsurgery.

Duffy's group also is evaluating the potential of SiMoA in neurological diseases.

"There are no blood-based tests for neurological diseases like Alzheimer's, as the disease-associated proteins are usually trapped in the cerebrospinal fluid [CSF] and only very low amounts cross the blood brain barrier into circulation," he told SciBX. "Currently, spinal taps are required to detect these proteins in CSF, which is much more inconvenient than a blood test. We think that there is the opportunity to use our approach to detect the same disease-associated proteins that could also be present in blood but at much lower concentrations."

Quanterix has filed multiple patents covering SiMoA. Duffy said certain nondiagnostic and nonprognostic applications are available for licensing and partnering.

Lou, K.-J. SciBX 3(23); doi:10.1038/scibx.2010.694

Published online June 10, 2010

\section{REFERENCES}

1. Rissin, D.M. et al. Nat. Biotechnol.; published online May 23, 2010; doi:10.1038/nbt. 1641

Contact: David C. Duffy, Quanterix Corp., Cambridge, Mass. e-mail: dduffy@quanterix.com

COMPANIES AND INSTITUTIONS MENTIONED

Pacific Northwest National Laboratory, Richland, Wash.

Quanterix Corp., Cambridge, Mass.

University of Pennsylvania, Philadelphia, Pa. 